\title{
Revisiting the Complexity of Finding Globally Minimum Energy Configurations in Atomic Clusters
}

\author{
By G. W. Greenwood \\ Dept. of Electrical \& Computer Engineering, Portland State University, \\ Portland, OR 97207 USA
}

Published in Zeitschrift für Physikalische Chemie Vol. 211, 105-114, 1999

\begin{abstract}
It has previously been proven that finding the globally minimum energy configuration of an atomic cluster belongs in the class of NP-hard problems. However, this proof is limited only to homonuclear clusters. This paper presents a new proof which shows finding minimum energy configurations for heteronuclear clusters is also NP-hard.
\end{abstract}

\section{Introduction}

Atomic clusters are aggregates of atoms held together by the same forces that cause, for example, phase transition from vapor to liquid, formations of crystals, etc. Cluster sizes range from as few as three atoms up to several hundred atoms. The physical and chemical characteristics of a cluster often varies with its size. In fact, even the addition of a single atom can result in an entirely different structure. Only by successively adding more and more atoms will a crystal-like structure eventually be produced and some knowledge of the condensed phase attributes be determined [1].

The study of atomic clusters has steadily been increasing over the past decade [2]. Of particular interest is the cluster conformation (structure) which has the lowest total internal energy $E$. Knowledge of this minimum energy conformation provides valuable clues relating to the chemical and physical properties of the cluster. Unfortunately, searching for the globally minimum energy state of a cluster has proven to be enormously difficult. Indeed, Wille and Vennik [3] showed that locating the globally minimum energy state of a cluster of identical atoms - the homonuclear case - belongs in the class of NP-hard problems. This means there is little hope of exactly solving the problem in finite time for even moderate cluster sizes.

The purpose of this paper is two-fold. First, it will be shown why existing homonuclear proofs, and work from other related problems, cannot be used for the heteronuclear case where not all of the atoms are identical. Secondly, a proof will be presented which does show solving the heteronuclear problem is NP-hard.

\section{Preliminaries}

The problem of finding this globally minimum energy structure is equivalent to optimizing $E$ with respect to variations in all $3 N-6$ degrees of freedom where $N$ is the cluster size. 
One method of solving this optimization problem is to explore the potential energy surface (PES) composed of all possible cluster conformations. Each point on this surface represents a unique spatial arrangement of the constituent atoms. This multidimensional surface is characterized by numerous local minima, each indicating an energetically stable structure. If it were possible to enumerate all of these minima - and the saddles that link them - we could, in principle, describe the dynamics of chemical reactions governed by that surface. Unfortunately, enumerating all minima is extremely difficult because of their large number. Berry [4] indicates the number of geometrically distinct minima tends to grow exponentially with $N$. Moreover, the number of permutational isomers grows factorially with $N$.

A number of general methods have been proposed for finding global minima on a PES, in particular, and on hypersurfaces in general. For example, Monte Carlo methods [5], eigenvector following [6], evolution computation techniques [7]-[9], lattice optimization/relaxation techniques [10], and PES deformation techniques [11, 12] have all been used with varying degrees of success. After formally defining the clustering problem, we will discuss some of these techniques in greater detail.

The potential energy function for a cluster of $N$ atoms is given by

$$
V\left(r^{N}\right)=\frac{1}{2} \sum_{\substack{i, j \\ i \neq j}}^{N} v\left(r_{i}-r_{j}\right)
$$

where $r^{N}=\left(r_{1}, r_{2}, \ldots, r_{N}\right), r_{i}$ is the position vector of the $i$ th atom and $v\left(r_{i}-r_{j}\right)$ is a function representing the pairwise interaction between atoms. Lennard-Jones or Morse functions are frequently used for these functions. Our optimization problem of interest is the Discrete Cluster Problem (DCP) which is formally defined as follows:円

\section{DISCRETE CLUSTER PROBLEM}

INSTANCE: Given finite number of points in real space, a distance $d(i, j) \in Z^{+}$between two points $i, j$, an integer $N \in Z^{+}$and a known potential energy function defined by (1). QUESTION: Is there a way to assign $N$ atoms to $N$ unique points so as to minimize the sum of their pairwise interactions?

The potential energy of a cluster actually equals the sum of $N$-body interactions. Restricting this sum to only two-body interactions provides only a qualitative approximation. Three-body interactions can be important, but are usually ignored in first order approximations [13. Although many-body interactions are needed for quantitative modeling, little is actually known about higher order terms. Many-body potential energy functions do exist [14, though in practice, only two-body terms are used for the sake of computational speed. Consequently, the discussion here will likewise be restricted to the two-body case.

It may appear that the large amount of work done with hard-sphere packing problems will be helpful in solving instances of DCP. Unfortunately, such is not the case because the objective of the two problems are quite different. Hard-sphere packings try to place $N$ spheres in Euclidean space so that all can, without overlap, fit within as small a volume as possible [15]. DCP deals with soft, compliant spheres which interact via pairwise interaction

\footnotetext{
${ }^{1}$ The definition given covers both homonuclear and heteronuclear systems.
} 
functions. Figure 1 shows a Lennard-Jones function, which is typical. Therein lies the major difference between hard-sphere and soft-sphere systems: the former has no preferred distance between the spheres while the latter does. Put another way, a hard-sphere packing algorithm attempts to minimize the interatomic distance $r$. Yet, a comparison with Figure 11 clearly shows this does not yield the lowest energy state for an atomic pair. Hard-sphere packing studies can thus be expected to provide little help.

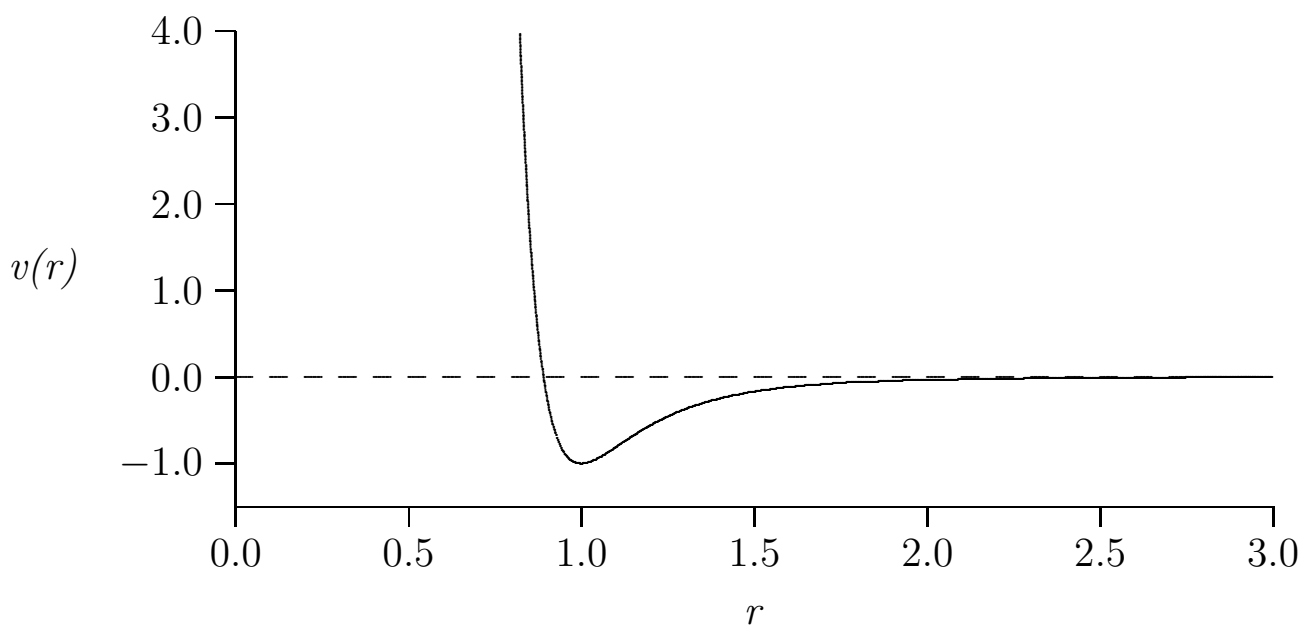

Figure 1: The scaled Lennard-Jones potential function $v(r)$. The scaled interatomic distance is denoted by $r$.

An algorithm that searches for solutions to the homonuclear version of DCP was recently proposed by Hendrickson [16. The general approach exploits structural information to decompose the global optimization problem into a set of smaller optimization problems. More specifically, $N$ objects in Euclidean space are represented as an undirected graph where the edge weights reflect the interobject distance. A subgraph is identified, the relative positions of the vertices are optimized, and the subgraph is then treated as a rigid body. These rigid bodies are ultimately combined to determine the overall structure. Such an approach won't work for the heteronuclear case, but this discussion will be differed until Section 3 .

Northby [10 performed a lattice based search that takes an initial conformation and allows it to relax to an energy minimum. Essentially, a random search is conducted to compile a list of isomers. After pruning any geometrically equivalent isomers, the remaining conformations are relaxed under a Lennard-Jones pairwise interaction function. While this technique will work with heteronuclear clusters, it does depend upon a gradient search which makes it susceptible to stopping at local optima.

Kostrowicki et al. 11], presented a technique that "deforms" the PES in such a way that the number of local minima is dramatically reduced. This deformation requires solving a partial differential equation called a diffusion equation. All atoms are assumed to interact by a Lennard-Jones type of function which is approximated by a linear combination of Gaussian functions to permit expressing the answer analytically. Solving these diffusion equations is not necessarily easy as there are some pathological conditions. For example, numerical 
problems can occur when the atoms are separated by large distances and so interact only weakly. This technique works best if one can suitably modify the boundary conditions for the diffusion equation, but care must be taken to ensure the minimum energy state is not removed from the deformed PES.

Wales and Doye [12 described another PES transformation technique called "basin hopping". Here the PES is converted into a set of basins of attraction for the local minima. This approach does has the advantage of not altering the energies of the minima. It has been used to find many of the local minima for Lennard-Jones cluster sizes up to $N=110$, The authors suggest some improvements that could be made to improve the efficiency of their approach.

It is important to note that none of these techniques will, with certainty, guarantee a successful search for the globally minimum energy configuration. In the next section we will see why solving instances of DCP has proven to be so difficult.

\section{Complexity in Homonuclear Clusters}

An instance of DCP can be solved by exploring the PES associated the cluster, and any algorithm that does this can be used with both homonuclear and heteronuclear systems. A number of researchers indicate the number of local optima in a PES grows exponentially with $N$ [4, 17, 18]. So, exactly how much effort is required to explore an exponentially large hypersurface? Some insight can be found from the theory of NP-completeness [19].

Suppose each time step a new point on the hypersurface is visited. Repeating this process until all points are visited - a procedure guaranteed to find the minimum energy state — will take an exponential number of time steps. Now consider an arbitrary NP-complete problem $\mathcal{P}$. If we found an algorithm that could solve $\mathcal{P}$ in polynomial time, then this algorithm could solve every NP-complete problem in polynomial time. Unfortunately, no algorithm that solves an NP-complete problem has ever been found which executes in less than an exponential number of steps. This suggests the effort required to conduct a blind search of the hypersurface is similar to the effort required to solve an NP-complete problem. NPcomplete problems are computationally intractable, which gives some idea of the difficulty one faces in solving DCP. (NOTE: This does not prove DCP is NP-complete. It merely shows the ramifications of having so many local optima.)

NP-complete problems are defined as decision problems, that is, problems answered by either 'yes' or 'no'. NP-hard problems ask for the optimal solution to an NP-complete problem. And, they have at least the same level of difficulty to solve as does the corresponding NP-complete problem. One particular NP-hard problem plays a pivotal role in the complexity analysis of DCP. It is the well known Traveling Salesman Problem (TSP) which is formally defined as follows:

\section{TRAVELING SALESMAN PROBLEM}

INSTANCE: A finite set $C=\left\{c_{1}, c_{2}, \ldots, c_{m}\right\}$ of cities, and a distance $d\left(c_{i}, c_{j}\right) \in Z^{+}$for each pair of cities $c_{i}, c_{j} \in C$.

QUESTION: What permutation

$$
\left[c_{\pi(1)}, c_{\pi(2)}, \ldots, c_{\pi(m)}\right]
$$


of $C$ will minimize the tour length

$$
\left\{\sum_{i=1}^{m-1} d\left(c_{\pi(i)}, c_{\pi(i+1)}\right)\right\}+d\left(c_{\pi(m)}, c_{\pi(1)}\right) ?
$$

Wille and Vennik [3] used TSP to prove solving a homonuclear instance of DCP is NPhard. It is worthwhile to examine their proof in some detail since our proof of NP-hardness for the heteronuclear case follows a similar line of reasoning.

Instances of DCP can be expressed in graph-theoretical terms using a graph $G=(V, E)$ with vertex set $V$ and edge set $E$. The graph $G$ is complete (i.e., the edge $e=\langle u, v\rangle \in$ $E \forall u, v \in V)$. Furthermore, $|V| \gg N$. The edges are assigned weights $w(e) \forall e \in E$ where the weights reflect the interaction between vertices. An instance of DCP is therefore equivalent to selecting $V^{\prime} \subset V$ with $\left|V^{\prime}\right|=N$ so that

$$
\begin{aligned}
& \frac{1}{2} \sum_{\substack{u, v \in V^{\prime} \\
e=\langle u, v\rangle}} w(e)=\text { minimal } \\
& \quad e=\left\langle u\left(\frac{1}{2}\right)\right.
\end{aligned}
$$

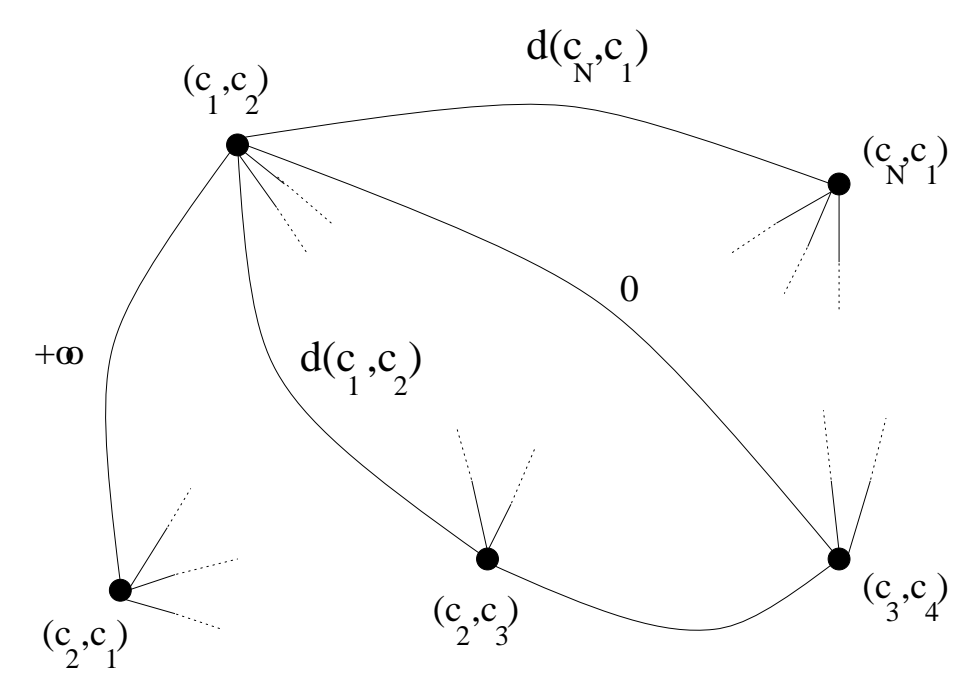

Figure 2: An example graph. The graph is dense and edges are weighted as given by Eq. (3). The problem is to select $N$ vertices so that the sum of the weighted edges is minimized.

The proof of Wille and Vennik uses an undirected graph $G=(V, E)$ with $|V|=N(N-1)$ vertices (see Figure 2). Each vertex is labeled with $\left(c_{i}, c_{j}\right)$ where $i, j=1, \ldots, N$ and $i \neq j$. This indicates city $c_{j}$ is visited immediately after city $c_{i}$. Edges are then unordered pairs of the form

$$
e=\left\langle\left(c_{i}, c_{j}\right)\left(c_{k}, c_{l}\right)\right\rangle
$$


with edge weights

$$
w(e)= \begin{cases}0 & \text { if } i \neq k, j \neq l \\
+\infty & \left\{\begin{array}{l}
\text { if } i=k, j \neq l \\
\text { if } j=l, i \neq k \\
\text { if } l=i, k=j
\end{array}\right. \\
d\left(c_{k}, c_{l}\right) & \text { if } l=i, k \neq j \\
d\left(c_{i}, c_{j}\right) & \text { if } k=j, l \neq i\end{cases}
$$

Selecting $N$ vertices, $\left\{\left(c_{\pi(i)}, c_{\pi(i+1)}\right) ; i=1, \ldots, N, c_{\pi(N+1)}=c_{\pi(1)}\right\}$, so that the sum of the weights is minimal is equivalent to finding a minimal length tour $\left\langle c_{\pi(1)}, c_{\pi(2)}, \ldots, c_{\pi(N)}\right\rangle$ thus solving an instance of TSP. By restriction [19], this also makes DCP NP-hard to solve. This completes the Wille and Vennik proof.

The weight assignments given in Eq. (3) require some clarification. In TSP, weight is equivalent to distance whereas in DCP weight is equivalent to pairwise potential energy. Any plausible solution to an instance of TSP can visit each city but one time. In valid tour moves, the edge weight equals the intercity distance. Disjointed tours have edges with zero weight - effectively removing that edge from the edge set $E$. This insures all tours can be defined as a permutation of $m$ cities (see below). An illegal tour move has an edge with infinite weight. The total weight of the edges traversed in a tour measures the "goodness" of that tour; good tours have lower total edge weights. More specifically,

1. $i \neq k$ and $j \neq l$. This defines a disjointed tour where the tour visits $c_{i}$ followed by $c_{j}$ and $c_{k}$ followed by $c_{l}$. However, it is not shown what other cities were visited between $c_{j}$ and $c_{k}$. Therefore, it is not possible to describe the permutation and compute its tour length.

2. $i=k$ and $j \neq l$. This defines an illegal tour that leaves the same city to visit two different cities.

3. $j=l$ and $i \neq k$. This defines an illegal tour where two distinct cities visit the same next city.

4. $l=i$ and $k=j$. This defines an illegal cyclic tour among only two cities.

5. $l=i, k \neq j$ or $k=j, l \neq i$. These are legal tours.

Each of the $N$ vertices in the minimal length tour is assigned a distinct atom from the cluster. In homonuclear clusters all one-to-one assignments of atoms to these $N$ vertices are equivalent since the edge weights are based only on interatomic distance. This begins to explain why the Wille and Vennik proof [3] and the Hendrickson algorithm [16] cannot be extended to the heteronuclear case.

First, consider a homonuclear cluster with $N>2$ atoms and suppose a new cluster is formed by swapping the spatial position of two atoms. It is not possible to tell any physical difference between the two clusters because all atoms are identical and the interatomic distances remain unchanged. Indeed, the new cluster will have a total energy identical to the original cluster because the pairwise interaction functions $\left(v\left(r_{i}-r_{j}\right)\right.$ in Eq. (1) $)$ remain unchanged. Now suppose the cluster is composed of two distinct atom types, say Ar and Xe. 
The repulsive and attractive forces experienced by an Ar-Ar atom pair differs from those experienced by Ar-Xe or Xe-Xe pairs even if all pairs are separated by the same interatomic distance [20]. Swapping atom positions in heteronuclear clusters changes the type of atoms which interact, altering the individual interaction functions, and giving a different total energy to the new cluster.

With homonuclear clusters it is acceptable to consider atoms as simple identical spheres where only interatomic distances contribute to the total energy. It is natural to model this system as an undirected graph where the edge weights reflect forces derived solely from the interatomic distances. The search algorithm from [16] takes this approach thereby permitting the cluster to be optimized in stages by optimizing the relative positions in subgraphs. The complexity proof given in [3] also made that assumption. In fact, the graph used for that proof was constructed specifically without requiring any pair type information to set the edge weights. That restriction was necessary to establish an equivalence between TSP and DCP.

In heteronuclear systems, both atom type and distance determine pairwise forces so the corresponding graph must have edge weights that take both distance and atom type into consideration. Even the relative positions of vertices from a subgraph cannot be optimized without the weights being set in this manner. Consequently, search algorithms such as 16 cannot be used for heteronuclear clusters because the overall cluster structure depends on connecting rigid bodies, formed from optimized subgraphs, where the edge weights only consider interatomic distance.

To apply the proof of Wille Vennik [3] to heteronuclear clusters, the graph would have to be augmented with additional vertices. For example, suppose in the original (homonuclear) graph two vertices $i$ and $j$ are connected by an edge $e=\langle i, j\rangle$. Then $w(e)$ is based solely on the interatomic distance between atoms $i$ and $j$. The augmented graph would have a new vertex $j^{\prime}$ where the added edge $e^{\prime}=\left\langle i, j^{\prime}\right\rangle$ has a weight $w\left(e^{\prime}\right)$ computed from the interaction of two dissimilar atoms, spaced at a distance $d\left(i, j^{\prime}\right)=d(i, j)$. However, now the mere selection of $N$ vertices - sufficient to solve a homonuclear DCP-does not guarantee the correct mixture of atom types present in the heteronuclear cluster. This restricts the proof from [3] to only homonuclear systems.

\section{Complexity in Heteronuclear Clusters}

A different proof of complexity is needed for the heteronuclear clusters. This new proof makes use of the following NP-hard problem [19]:

TRAVELING SALESMAN EXTENSION (TSE)

INSTANCE: A finite set $C=\left\{c_{1}, c_{2}, \ldots, c_{m}\right\}$ of cities, a distance $d\left(c_{i}, c_{j}\right) \in Z^{+}$for each pair of cities $c_{i}, c_{j} \in C$, a bound $B \in Z^{+}$, and a "partial" tour

$$
\Theta=\left\langle c_{\pi(1)}, c_{\pi(2)}, \ldots, c_{\pi(K)}\right\rangle
$$

of $K$ distinct cities from $C, 1 \leq K \leq m$.

QUESTION: Can $\Theta$ be extended to a full tour

$$
\left\langle c_{\pi(1)}, c_{\pi(2)}, \ldots, c_{\pi(K)}, c_{\pi(K+1)}, \ldots, c_{\pi(m)}\right\rangle
$$


having total length $B$ or less?

Consider a heteronuclear cluster which has a single atom of one type (denoted by $\alpha$ ) and $N-1$ atoms of a different type (denoted by $\beta$ ). A completely connected graph is $G=(V, E)$ with $|V|=N(N-1)$ is constructed with each vertex labeled as described in Section 3. Without loss in generality preassign an $\alpha$-atom to all vertices with labels $\left(c_{1}, c_{j}\right)$, $j=2, \ldots, N$ and preassign a $\beta$-atom to all remaining vertices.

The search begins with a scan of all edges that touch vertices with $\alpha$-atom assignments. Select the minimum weight edge. This edge defines a partial tour $\left\langle c_{\pi(1)}, c_{\pi(2)}\right\rangle$. Now select $N-2$ more vertices, $\left\{\left(c_{\pi(i)}, c_{\pi(i+1)}\right) ; i=3, \ldots, N, c_{\pi(N+1)}=c_{\pi(1)}\right\}$, so that the sum of these weights is minimal. (This search is limited to vertices with $\beta$-atom assignments in order to maintain the proper mixture of atom types in the corresponding cluster.) Finding a minimal length full tour is thus equivalent to solving an instance of TSE. This proves that DCP is NP-hard to solve for heteronuclear clusters as well.

\section{Discussion}

The complexity in solving DCP forces researchers to use heuristic search algorithms. Hillclimbing algorithms are not expected to do well because the PES has many local optima and it is highly likely the algorithm will quickly stop at one of them. Conversely, stochastic search algorithms can be quite effective in such multimodal hypersurfaces. It is therefore natural to ask if any one particular algorithm stands out as doing especially well against DCP.

This question is not easy to answer and we need to turn to the No Free Lunch (NFL) Theorem [21] for an explanation. Essentially, this theorem says if some optimization (search) algorithm performs particularly well over a certain class of optimization problems, then it most likely will not perform as well over all remaining optimization problems. This means one cannot choose, for example, simulated annealing to use for DCP just because it happens to work well for, say, scheduling problems. Direct comparisons between algorithms are insightful. But, without a conscientious attempt to make the comparisons fair, the results may be inconclusive, or in the worst case, be completely wrong [22]. Monte Carlo techniques have been dominant in the area of cluster studies, which is why newly proposed search algorithms are normally compared against them.

Most notable among the new algorithms are the evolutionary algorithms, which conduct searches that mimic Darwinian evolution: a "population" of clusters evolve to a low energy state by altering existing configurations via stochastic reproduction operators. Natural selection determines which configurations survive to undergo further reproduction operations.

Comparisons between evolutionary algorithms and Monte Carlo techniques have favored the former, although such comparisons sometimes lack sufficient mathematical rigor. For example, Zeiri 9] concluded that a genetic algorithm converges faster than simulated annealing after comparing the average of five runs - an unusually small sample size. Normally results should be averaged over a considerably higher number of runs to help remove any potential bias in the random number generators used in the algorithms. Nevertheless, there

\footnotetext{
${ }^{2}$ As before, edges with weight 0 are effectively removed from the graph.
} 
is growing empirical evidence that says evolutionary algorithms consistently outperform the

Monte Carlo techniques when applied against DCP [8] 20] 223]-26]. It appears as though evolutionary algorithms would be a good first choice search algorithm for cluster studies.

\section{Acknowledgement}

The author wishes to thank the anonymous reviewers who made several pertinent and valuable suggestions.

\section{References}

[1] Z. Bačić and R. Miller, J. Phys. Chem. 100 (1996) 12945.

[2] Cluster Ions, C. Ng. T. Baer and I. Powis (Eds.), John Wiley \& Son, New York (1993) and references therein

[3] L. T. Wille and J. Vennik, J. Phys A. 18 (1985) L419.

[4] R. S. Berry, Chem. Rev. 93 (1993) 2379.

[5] T. Pang, Chem. Phys. Lett. 228 (1994) 555.

[6] A. Banerjee, N. Adams, J. Simons and R. Shepard, J. Phys. Chem. 89 (1985) 52.

[7] G. Greenwood and Y. Liu, Proc. of EP98, (1998)

[8] B. Hartke, J. Phys. Chem. 97 (1993) 9973.

[9] Y. Zeiri, Phys. Rev. E 51 (1995) R2769.

[10] J. A. Northby, J. Chem. Phys. 87 (1987) 6166.

[11] J. Kostrowicki, L. Piela, B. Cherayil, and H. Scheraga, J. Phys. Chem. 95 (1991) 4113.

[12] D. Wales and J. Doye, J. Phys. Chem. A 101 (1997) 5111.

[13] M. Klein and L. Lewis, Chem. Rev. 90 (1990) 459.

[14] S. Erkoc, Phys. Rpts. 278 (1997) 79.

[15] I. Stewart, Sci. Amer. 266 (1992) 112.

[16] B. Hendrickson, SIAM J. Opt. 5 (1995) 835.

[17] M. R. Hoare, Adv. Chem. Phys. XL (1979), 49.

[18] K. D. Ball, R. Berry, R. Kunz, F. Li, A. Proykova and D. Wales, Science 271 (1996) 963. 
[19] M. R. Garey and D. S. Johnson, Computers and Intractability: A Guide to the Theory of NP-Completeness, W. H. Freeman \& Co., NY, (1979)

[20] W. J. Pullan, J. Comp. Chem. 18 (1997) 1096.

[21] D. H. Wolpert and W. G. Macready, IEEE Trans. on Evolutionary Comp. 1 (1997) 67.

[22] G. Greenwood, ACM Software Engr. Notes 22 (1997) 92.

[23] G. Greenwood, Tech. Rpt. TR/97-08, Dept. of Comp. Sci., Western Michigan Univ. (1997)

[24] D. M. Deaven and K. M. Ho, Phys. Rev. Lett. 75 (1995) 288.

[25] J. A. Niesse and H. R. Mayne, J. Chem. Phys. 105 (1996) 4700.

[26] D. M. Deaven, N. Tit, J. R. Morris and K. M. Ho, Chem. Phys. Lett. 256 (1996) 195. 